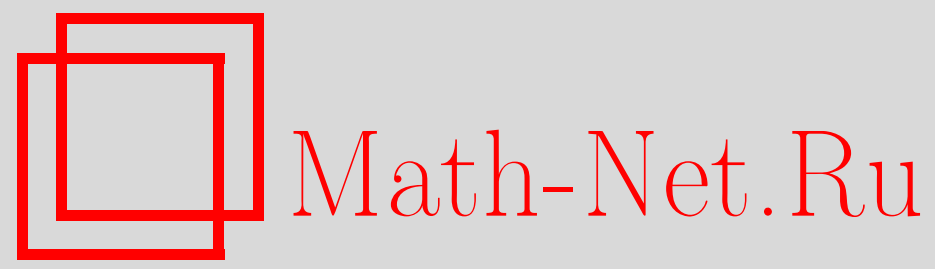

С. П. Новиков, Алгебраическая топология, Совр. пробл. матем., 2004, выпуск 4, 3-45

DOI: https://doi.org/10.4213/spm7

Использование Общероссийского математического портала MathNet.Ru подразумевает, что вы прочитали и согласны с пользовательским соглашением

http://www.mathnet.ru/rus/agreement

Параметры загрузки:

IP : 52.87 .193 .239

26 апреля 2023 г., 14:02:22

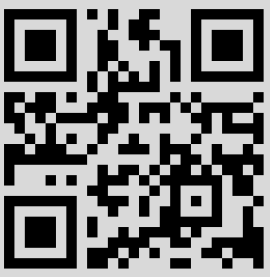




\title{
Алгебраическая топология
}

\author{
С. П. Новиков
}

Алгебраическая топология (А.Т.) - область математики, возникшая для изучения таких свойств геометрических фигур (в широком смысле - любых объектов, где можно говорить о непрерывности) и их отображений друг в друга, которые не меняются при непрерывных деформациях (гомотопиях). В принципе целью А.Т. является полное перечисление таких свойств. Само название А.Т. происходит от определяющей роли алгебраических понятий и методов при решении задач этой области. Наиболее фундаментальными классами объектов, свойства которых изучаются в А.Т., являются: комплексы (многогранники, полиэдры) симплициальные, клеточные и другие; многообразия - замкнутые, открытые, с краем (границей), подразделяющиеся в свою очередь на гладкие (дифференцируемые), аналитические, комплексно-аналитические, кусочно-линейные и, наконец, чисто непрерывные (топологические); косые произведения (расслоения) и их сечения. Основные типы отображений, рассматриваемые в А.Т., - это произвольные непрерывные, кусочно-линейные и гладкие отображения или их подклассы: гомеоморфизмы, в частности непрерывные, кусочно-линейные или гладкие (диффеоморфизмы); вложения одного объекта в другой, а также погружения (локальные вложения, иммерсии). Важнейшим понятием А.Т. является понятие деформации. Деформации подвергается отображение какого-то класса одного объекта в другой. Основными типами деформаций являются: гомотопия или произвольная непрерывная (гладкая, кусочно-линейная) деформация непрерывного (гладкого, кусочно-линейного) отображения; изотопия (непрерывная, гладкая, кусочно-линейная) - т.е. деформация

(C) Новиков С. П., 2004 
гомеоморфизма, вложения или погружения, где в процессе деформации в каждый момент времени отображение остается гомеоморфизмом, вложением или погружением. Главные внутренние проблемы А.Т. - это проблемы классификации многообразий относительно гомеоморфизмов (непрерывных, гладких, кусочно-линейных), классификация вложений (погружений) относительно изотопий (регулярных гомотопий), классификация общих непрерывных отображений относительно гомотопий. Важную роль в решении этих задач играет классификация комплексов и многообразий относительно так называемого гомотопического типа или гомотопической эквивалентности.

Богатство идей, внесенных топологией, теми крупнейшими учеными, которые в ней работали, поставило эту область в центр мировой математики, начиная с середины XX века. Например, с 1950 до 2002 года активным математикам, признанным лучшими и не превышающим 40 лет, было присуждено в общей сложности 44 Медали Филдса на Всемирных Математических Конгрессах. Среди них имена Серра (1954), Тома (1958), Милнора (1962), Атьи (1966), Смейла (1966), Новикова (1970), Квиллена (1978), Тёрстона (1982), Дональдсона (1986), Фридмана (1986), Виттена (1990), Джонса (1990), Концевича (1998), центральная часть математического вклада которых в те годы относилась к топологии, а также имена Кодаиры (1950), Гротендика (1966), Мамфорда (1974), Делиня (1978), Яо (1982), Воеводского (2002), работавших на стыке идей топологии, алгебраической геометрии и гомологической алгебры. Как видно, с 1954 по 1970 годы около половины Медалей Филдса была присуждена топологам, оказавшим влияние на много других областей математики. Хотя мы и знаем один-два огорчительных случая, когда ученому высшего класса и подходящего возраста не была присуждена Медаль Филдса, но в целом приведенная статистика правильно отражает положение топологии на вершине математики в определенный период.

Исторический очерк: топологические идеи до XIX века. Наивное понимание грубых топологических различий между трехмерными геометрическими фигурами существовало уже в глубокой древности. Всякому очевидно, что число дыр или ручек в трехмерной области (фигуре) не изменится, если ее гнуть без разрывов и самопересечений. Сложность узлов, сделанных из корабельных веревок, привлекла внимание античных греков. 
Однако первые топологические наблюдения в форме точных математических соотношений и теорем возникли лишь в XVIII веке у Л. Эйлера: число вершин минус число ребер плюс число граней выпуклого многогранника равно 2; как открыл позднее Пуанкаре, подобная альтернированная сумма является топологическим инвариантом любого комплекса. Задача о трех домах и трех колодцах: доказать, что три дома нельзя соединить с тремя колодцами путями, не пересекающими друг друга. На современном языке, этот граф (одномерный комплекс) с шестью вершинами и девятью ребрами нельзя вложить в плоскость без самопересечений.

Топологические идеи в XIX веке. До XIX века такие наблюдения носили лишь характер игрушек вроде оригинальных олимпиадных задач, порожденных игрой чистого ума - подобно многим задачам теории чисел. Во второй четверти XIX века ситуация изменилась: Гаусс пришел к ряду нетривиальных топологических наблюдений после анализа опытов Фарадея, где человечество впервые увидело электромагнитные явления. В частности, Гаусс открыл так называемое число защепления двух замкнутых попарно непересекающихся кривых в трехмерном пространстве, не меняющихся при деформациях без пересечений. Именно Гаусс и поставил задачу о построении точной теории подобных свойств. Термин "топология" возник в работе его ученика Листинга. Топологическую теорию двумерных многообразий сильно продвинул Риман ("римановы поверхности"). Вообще, двумерная топология, по существу, возникла как важнейшая сторона нового тогда комплексного анализа - в трудах Коши на плоскости и в трудах Абеля, Якоби и Римана на двумерных многообразиях с нетривиальной топологией. Ряд топологических наблюдений был сделан физиками: Кельвин интересовался узлами. Он исходил из любопытных свойств замкнутых вихревых линий, открытых им в гидродинамике, и хотел применить узлы для классификации атомов (мы знаем, что это оказалось ложной идеей). Его ученик Тейт первым начал систематически развивать теорию узлов, высказал интересные гипотезы в конце XIX века, доказанные лишь недавно. Максвелл обратил внимание на соотношение между числами критических точек функций разных индексов: для изолированного острова число ям минус число перевалов плюс число вершин равно 1. Это отдаленный прообраз идей “теории Морса". Пуанкаре начал последовательно применять топологические идеи для анализа качественного поведения траекторий динамических систем, 
особенно для созданной им теории систем на плоскости. Им же топология была выделена в отдельную область математики, которую он назвал "Анализ Ситус". В числе наиболее простых и фундаментальных топологических характеристик оказались обобщения числа дыр и ручек: это числа Бетти с номером $k$ (числа в определенном смысле независимых $k$-мерных циклов в исследуемом пространстве, области или многообразии). Так как Бетти, согласно топологическим легендам, был химиком, то эти характеристики и их дальнейшие обобщения получили название "гомологий". Пуанкаре дал топологическую классификацию двумерных многообразий. Он ввел важнейший топологический инвариант фундаментальную группу пространства, состоящую из гомотопических классов замкнутых путей с началом и концом в одной общей точке - и построил топологическую теорию накрытий. Им открыт закон двойственности Пуанкаре, утверждающий, что для замкнутых $n$-мерных многообразий числа Бетти определенных типов с номерами $k$ и $n-k$ совпадают. Проблема классификации трехмерных многообразий встретила большие трудности: до самого последнего времени не удавалось доказать, что всякое односвязное трехмерное многообразие (где фундаментальная группа единична) гомеоморфно сфере. Это гипотеза Пуанкаре.

Топология в XX веке: очерк до 1945 г. В начале XX века эта область стала называться топологией. В 30-х годах, когда алгебраические методы приобрели решающее значение, эта область, благодаря С. Лефшецу, стала называться алгебраической mопологией. В течение XX века в развитие алгебраической топологии и ее приложений внес вклад ряд крупнейших математиков. В 1910-х годах Брауэр доказал, что размерность многообразий - инвариант непрерывных гомеоморфизмов; он открыл степень отображения сфер на сферы и теорему существования неподвижной точки отображений диска в себя. В начале XX века Ден далеко продвинул идеи трехмерной топологии. Исходя из фундаментальной группы, он создал комбинаторную теорию конечно определенных групп, сформулировал ее основные алгоритмические проблемы: проблему тождества слов, сопряженности и изоморфизма. Сомнений в том, что эти свойства являются алгоритмически распознаваемыми, в те времена не было.

В 1920-х годах Александер доказал топологическую инвариантность гомологий (чисел Бетти). Оказалось, что гомологии являются более грубыми инвариантами гомотопического типа, от- 
крытого Александером. Вьеторис, П. Александров и Чех распространили теорию гомологий на общие пространства. Э. Нётер высказала важную идею, способствовавшую полной алгебраизации теории гомологий. С. Лефшец создал глубокую теорию гомологий алгебраических комплексных проективных многообразий, перенес ее идеи в топологию, особенно теорию пересечений циклов. Э. Картан, исходя из идей Пуанкаре и римановой геометрии, ввел дифференциальные формы и теорию гомологий на их основе ("тензорная теория гомологий"). Высказанная им гипотеза, что эта теория эквивалентна обычной топологии, была в 30-х годах доказана его учеником де Рамом. Эта теория стала называться "теорией де Рама". В 1920-х годах Морс открыл топологические методы вариационного исчисления, топологические неравенства Морса для чисел критических точек ("теория Морса"). Около 1930 г. Люстерник и Шнирельман развили весьма своеобразный вариант теории типа Морса для вырожденных критических точек, особенно глубокий для двумерного случая; они доказали гипотезу Пуанкаре о трех геодезических: на римановом многообразии, гомеоморфном двумерной сфере, всегда существует не менее трех несамопересекающихся замкнутых геодезических. Важнейшие результаты Александера, Лефшеца, Морса и Люстерника-Шнирельмана не были математически строго доказаны. Теория Александера гомотопически инвариантной сингулярной теории гомологий была строго обоснована в 40-х гг. Эйленбергом и др., теория гомологий Лефшеца алгебраических многообразий - в 50-х гг., а результаты Люстерника-Шнирельмана о трех геодезических - лишь к началу 90-х гг.

В 30-х годах развитие алгебраической топологии стало еще более интенсивным. Возникла идея гомотопических групп (классов отображений сферы в пространство, где образ одной точки фиксирован), введенная Гуревичем. Еще ранее Х. Хопф нашел гомотопическую классификацию отображений $n$-мерных многообразий в $n$-мерную сферу: классы определяются степенью отображения. Он открыл позднее нетривиальность высших гомотопических групп сфер ("инвариант Хопфа"). Проблема вычисления гомотопических групп сфер стала в дальнейшем одной из центральных проблем алгебраической топологии - Понтрягин и Фрейденталь начали процесс ее решения. В частности, Понтрягин пришел отсюда к проблеме кобордизма: каковы алгебротопологические условия того, что замкнутое многообразие явля- 
ется границей многообразия с краем? Уже двумерная проективная плоскость не является границей ничего.

Алгебраизация различных вариантов законов двойственности типа Пуанкаре для гомологий в многообразиях (законы двойственности Александера и Лефшеца) привела к теории характеров для непрерывных групп (Понтрягин), а также к понятию "когомологий" (Колмогоров и Александер, 1935), двойственных к гомологиям. Позднее в 1940-х годах двойственность была глубоко осмыслена в рамках стабильной теории гомотопий ("двойственность Спеньера-Уайтхеда"). Когомологии оказались суперкоммутативным градуированным кольцом; операция умножения Колмогорова-Александера, ошибки в определении которой были исправлены Чехом и Уитни в 1936/37 г., идейно заимствованная из теории пересечений Лефшеца в алгебраической геометрии и теории Э. Картана в тензорном анализе, оказалась исключительно важной в алгебраической топологии. Важные сдвиги были осуществлены и в маломерной топологии: Нильсен и Магнус создали теорию автоморфизмов поверхностей, начали новые главы комбинаторной теории групп исходя из топологии, развитую ван Кампеном. Александер и Радемайстер развили теорию узлов и зацеплений замкнутых кривых в трехмерном пространстве. Были открыты такие важные инварианты узлов, как полином Александера, создана комбинаторная модель Радемайстера теории узлов (движения Радемайстера для плоских диаграмм узлов и зацеплений). Появилась теория кос $\vartheta$. Артина, по-видимому просто вычислившего фундаментальную группу пространств полиномов с некратными корнями (но не упомянувшего это). Появилось сведение теории узлов к косам (модель АлександераМаркова-младшего, строгое обоснование которой было опубликовано только в 80-х годах). Радемайстер открыл знаменитый комбинаторный инвариант трехмерных многообразий - так называемое "кручение Радемайстера" - решающий проблему классификации линзовых многообразий. Эта величина гомотопически неинвариантна. Уайтхед создал теорию "простого гомотопического типа" для этого феномена, сидящего на фундаментальной группе. Здесь впервые была выявлена глубокая интерференция фундаментальной группы с теорией гомологий, возникло расширение понятия детерминанта линейных преобразований. Это привело к теории обобщенного детерминанта матриц над кольцами, после осмысления и развития этой теории Уайтхедом 
в 40-х гг. (независимо к родственным идеям в чистой алгебре пришел Дьедонне, но без топологических применений), позднее привело к глубоким открытиям в анализе.

Одним из крупнейших общематематических достижений топологии 30-х годов было создание теории дифференцируемых многообразий и их вложений в евклидово пространство на базе идей трансверсальности гладких отображений (Уитни). Новый метод позволил построить вполне строгий аналитический и геометрический подход к построению и вычислению топологических инвариантов (например, теории пересечений циклов), основанный на трансверсальности и приведении в общее положение, неоценимый с точки зрения будущего анализа. Этот подход был развит Понтрягиным для вычисления первых нетривиальных $k$-мерных гомотопических групп $k$-мерной сферы $(k=n+1$ и $k=n+2$; для $k=n+2$ Понтрягин ошибся, не заметив так называемого "Arf-инварианта"; он исправил эту ошибку в 1950). Этот метод приводит к проблеме кобордизмов $k$-мерных многообразий, как уже упоминалось. Уитни, Штифель, Понтрягин и Черн открыли в конце 30-х годов важнейшие топологические инварианты гладких и комплексных многообразий - характеристические классы, связывающие воедино топологию, риманову геометрию и комплексный анализ. Это классы Штифеля-Уитни во всех когомологиях по модулю 2 и классы Понтрягина в целочисленных $4 k$-мерных когомологиях гладких многообразий; классы Черна лежат в $2 k$-мерных целочисленных когомологиях комплексных многообразий. Имеется по одному базисному классу каждого из указанных типов в каждой из указанных размерностей. Характеристическим классом считается также любой полином от базисных в кольце когомологий. Согласно теореме Понтрягина, для замкнутых многообразий - границ многообразий с краем - все характеристические вычеты modulo 2 Штифеля-Уитни, а также и числа Понтрягина в ориентируемом случае (т.е. классы размерности многообразия) равны нулю. Для проективной плоскости это не так, например.

Большое влияние на развитие топологии и смешанных дисциплин оказали результаты, посвященные исследованию проблемы неподвижных точек отображений многообразий в себя: возникновение формулы Лефшеца для алгебраического числа таких точек и теория Смита, изучающая топологические инварианты множеств неподвижных точек преобразований конечного порядка. 
Здесь были развиты глубокие алгебраические методы, дающие начало нетривиальной гомологической алгебры: стало необходимо разобраться в громадном количестве связей различных видов гомологий или гомологий различных объектов друг с другом. Уже здесь возникли простейшие операции в когомологиях с конечным полем коэффициентов, не сводимые к структуре кольца когомологий - кограничные операторы Бокштейна.

Алгебраическая топология в XX веке: 1945-1955 гг. К середине 40-х годов уже была создана теория расслоенных пространств и характеристических классов (упоминавшихся выше), была создана гомологическая теория препятствий к построению и продолжению отображений и сечений расслоений. Затем появились нетривиальные когомологические операции, не сводимые к умножению - квадраты и степени Понтрягина и Стинрода, операции Масси. Особенно важными оказались операции Стинрода в когомологиях modulo $p$ для всех простых чисел $p \geqslant 2$, вместе с простейшим оператором Бокштейна. Это линейные операторы, действующие в когомологиях всех пространств, коммутирующие с непрерывными отображениями (как говорят - "эндоморфизмы функтора когомологий"). Решая задачу Хопфа о вычислении когомологий асферичных пространств (у которых универсальная накрывающая стягиваема), Эйленберг и Маклейн основали то, что было названо "гомологической алгеброй" - теорию гомологий групп - в 1945 г. Независимо (но несколько позже) к подобным идеям из соображений чистой алгебры и алгебраической теории чисел пришел Д. Фаддеев. Эйленберг и Маклейн построили также алгебраическую модель пространств ("комплексы Эйленберга-Маклейна"), у которых все гомотопические группы, кроме одной, равны нулю. Этот цикл идей был завершен около 50 г. Постниковым, построившим обобщение этой модели на произвольные пространства ("система Постникова"). Принципиально, система Постникова дает полную информацию о гомотопическом типе комплексов и многообразий. В важном частном случае односвязных четырехмерных многообразий Понтрягин и Уайтхед решили эту задачу без систем Постникова в конце 1940-х гг. $\mathrm{X}$. Хопфом была установлена замечательная связь некоторых величин в гомотопических группах сфер (инварианта Хопфа) с известной проблемой алгебры: существуют ли вещественные алгебры с делением в размерностях $n \neq 1,2,4,8$, где мы знаем вещественные и комплексные числа, кватернионы и алгебры Кэли. 
Хопф, используя алгебру операций Стинрода, доказал, что таких алгебр нет для $n \neq 2^{q}$, но для $q>3$ это доказать не удавалось. Алгебраические соотношения между операциями Стинрода для $p=2$, найденные Адемом в конце 40-х гг., как указал Хопф, приводят к результату для $n \neq 2^{q}$. Сложилась такая ситуация, что в топологии было открыто большое количество нетривиальных алгебраических величин и законов связи между ними: фундаментальная группа и высшие гомотопические группы как модули (и даже супералгебра Ли-Уайтхеда, образуемая ими), группы гомологий и кольца когомологий, характеристические классы многообразий и расслоений, операции Стинрода и многое другое. Как их вычислять? В некоторых случаях удавалось вычислить гомологии пространств, используя триангуляции и новые для 40-х годов клеточные разбиения, введенные первоначально для обоснования теории Морса. Иногда удавалось вычислить, например, гомологии групп Ли и пространств петель, используя вариационное исчисление (теорию Морса), иногда помогала теория де Рама, где еще Э. Картан для однородных пространств использовал теорию групп Ли для подобных целей, сводя вычисление гомологий к инвариантным замкнутым формам. Таким образом, уже в 40-х гг. вычисление гомологий казалось обозримым, в то же время как проблема вычисления гомотопических групп в ряде случаев например для сфер - казалась трансцендентно трудной.

Во второй половине 40-х годов-начале 50-х гг. Лере, Серр, А. Картан, Том, Борель создали и успешно применили цикл замечательных алгебраических методов для вычисления гомологий расслоенных пространств, основанных на так называемых "спектральных последовательностях Лере". Это выдающееся открытие и породило то, что сейчас называется нетривиальной гомологической алгеброй, ее основным методом, позволяющим регулярным образом вычислить такие величины, которые ранее, как уже говорилось, считались в топологии трансцендентными. Это вычисление идет через посредство серии вспомогательных обобщенных "расслоений Серра", где работают спектральные последовательности Лере, шаг за шагом. Серр создал метод, позволивший вычислить все гомотопические группы сфер, пренебрегая конечными частями: они все конечны, кроме специальных случаев, групп размерности $4 n-1$ для $2 n$-сфер, где возникает инвариант Хопфа. Общая теорема Картана-Серра завершает такое же вычисление для всех тех пространств, чьи коль- 
ца когомологий пространства петель над полем характеристики нуль известны. Для развития методов вычисления конечных частей гомотопических групп, Сepp (для $p=2$ ) и А. Картан (для $p>2$ ) вычислили все алгебры Стинрода когомологических операций, упоминавшихся выше. Эти алгебры порождены операторами Стинрода и Бокштейна. Их элементы - это линейные операторы, действующие в когомологиях всех пространств и коммутирующие с непрерывными отображениями. Между ними имеются соотношения Адема-Картана - и только. Уже к концу 40-х годов стало ясно, что теория когомологий полностью определяется простым набором функториальных свойств ("аксиомы Эйленберга-Стинрода"), в которые входят: 1) "гомотопическая инвариантность"; 2) "функториальность" (ковариантность при отображениях пространств); 3) "точная последовательность пары"; 4) "аксиома вырезания"; и, наконец, 5) "нормировка" - все гомологии, кроме нульмерной, равны нулю для точки. Гомотопические группы - это более сложный функтор, где не выполняется аксиома вырезания, хотя аналогия между ними, называемая "двойственностью Экмана-Хилтона", в ряде случаев очень полезна. Алгебра Стинрода - это эндоморфизмы функтора когомологий, классификация которых сводится, согласно лемме Серра, к вычислению когомологий комплексов Эйленберга-Маклейна. Последняя задача и была в нужных пределах решена Серром $(p=2)$ и А. Картаном $(p>2)$. А. Борель провел серию глубоких вычислений для однородных пространств и установил глубокие алгебраические свойства спектральных последовательностей. А. Борель ввел в 1954 г. важное понятие "алгебр Хопфа": согласно теореме Хопфа, алгебра когомологий над полем характеристики нуль любого пространства с непрерывным умножением, обладающим единицей, является свободной суперкоммутативной алгеброй, т.е. тензорным произведением внешней алгебры на алгебру полиномов. Как указал Борель, доказательство Хопфа основывается на чисто алгебраических свойствах этих алгебр, которые он формализовал и назвал этот класс алгебр "алгебрами Хопфа", обобщив затем теорему Хопфа на поля конечной характеристики, где алгебра Хопфа может и не быть свободной. Значение для топологии теории алгебр Хопфа над конечными полями и гомологической алгебры для них стало ясно вскоре. Алгебры Хопфа вошли в чистую алгебру много позже. Впоследствии они стали общепризнаны и в квантовой математической физике, и в анализе. Том, 
развивая идеи трансверсальности и метод Понтрягина, красиво свел исследование проблем кобордизмов (выше) к задаче вычисления гомотопических групп вспомогательных "пространств Тома" (в частном случае Понтрягина это сфера). К ним же сводится, по схеме Тома, задача о реализации циклов гладкими подмногообразиями, и проблема "Стинрода" о реализации цикла как образа гладкого многообразия. В неориентируемом случае Том красиво решил эти задачи методом Серра. Верна теорема, обратная к уже упоминавшейся теореме Понтрягина: зануление вычетов Штифеля-Уитни достаточно для того, чтобы гладкое замкнутое многообразие было краем. Все циклы modulo 2 реализуются как образы многообразий. Для ориентируемого случая Том установил, что обратная к Понтрягину теорема верна лишь с точностью до кратности. Проблема Стинрода разрешима также лишь для кратного цикла. Этим же методом "комплексов Тома", с помощью квадратов Стинрода для $p=2$, Том доказал гомотопическую инвариантность классов Штифеля-Уитни, а Ву полностью эффективно вычислил их методом Тома (формулы Ву).

Любопытно сравнить это с наиболее глубокими результатами вычисления гомотопических групп сфер и кобордизмов, полученных прямым геометрическим методом Понтрягина (Рохлин): он установил в начале 50-х гг., что $(n+3)$-мерная гомотопическая группа $n$-мерной сферы для $n \geqslant 5$ изоморфна конечной циклической группе порядка либо 12 , либо 24 , но ошибочно утверждал сначала, что порядок равен 12, пока не появилась работа Серра. Рохлин доказал также, что любое трехмерное многообразие - граница четырехмерного (т.е. кобордантно нулю), а любое четырехмерное ориентируемое замкнутое гладкое многообразие кобордантно конечному объединению комплексных проективных плоскостей. Рохлин открыл новый важнейший инвариант ориентируемого кобордизма - так называемую "сигнатуру" многообразия (разность чисел положительных и отрицательных квадратов матрицы пересечений $2 k$-циклов в $4 k$-мерном многообразии (ориентируемом)). Из результатов Рохлина для $k=1$ и Тома для всех $k \geqslant 1$ вытекает, что сигнатура выражается линейно через числа Понтрягина. Красивая формула была получена Хирцебрухом в 1954 г., блестяще применившим этот результат к доказательству общей теоремы Римана-Роха в алгебраической геометрии. Значение формулы сигнатуры для многих целей ярко проявилось в будущем развитии математики. Полные доказательства 
теорем Рохлина были опубликованы лишь в конце 50-х гг; прямой геометрический вывод того, что упомянутая гомотопическая группа $n$-сферы имеет порядок 24 , а не 12 , был опубликован лишь в 70-х гг., когда это стало важно для маломерной топологии. Анализ причин, ведущих к порядку 24 , а не 12 , привел Рохлина к примеру 2-цикла в односвязном 4-многообразии, не реализующимся гладко вложенной 2-сферой. Сам этот результат им не публиковался: он был упомянут со ссылкой на Рохлина у МилнораКервера в начале 60-х гг. Проблема оптимальной гладкой реализации 2-циклов поверхностью наименьшего рода в односвязном 4-многообразии стала позднее широко известна как "проблема Тома", решенная уже в 90-х гг. совсем другими методами (пришедшими из квантовой теории поля). Конечность гомотопических групп сфер приводится по схеме Дольда к следствию, что классы Понтрягина не являются гомотопическими инвариантами замкнутых многообразий (Дольд, 1956). Для этого результата достаточно уже конечности гомотопических групп размерности $(n+3)$ для $n$-мерной сферы. Для односвязных многообразий нет никаких гомотопически инвариантных соотношений между интегралами от классов Понтрягина по циклам, кроме формулы Хирцебруха для сигнатуры. В неодносвязных многообразиях гипотеза Новикова (ниже) описывает все гомотопически инвариантные интегралы. Она доказана в ряде случаев.

Таким образом, в результате периода грандиозного идейного подъема 1945-1955 гг. был создан уникальный ансамбль методов, позволивший А.Т. достичь своего самого высшего уровня в самом центре мировой математической мысли. На этом уровне она и держалась еще примерно 15 лет, до начала 70 -х гг. К описанию периода наивысшего расцвета классической А.Т. мы сейчас перейдем.

А.Т. в период 1956-1970 гг. Начало нового периода определяется знаменитым открытием Милнора нестандартных гладких структур на 7-сфере (1956). Вы смотрите на аналог известного расслоения Хопфа, где 3-сфера расслоена по окружности с базой 2 -сферой. В семимерном аналоге базой является 4-сфера, слой - это 3-сфера, а все пространство - 7-сфера, судя по гомотопическому типу. Как указывает теория расслоений, в отличие от случая Хопфа, здесь имеется бесконечная серия подобных расслоений: меняется первый класс Понтрягина, которого не было у обычного расслоения Хопфа. Все эти семимерные многообра- 
зия являются очевидной границей восьмимерных - расслоений над 4-сферой со слоем 4-диск, с разными классами Понтрягина, но общим кольцом когомологий. Если предположить, что край - это обычная 7-сфера, то ее можно заклеить диском и получить серию гладких многообразий, сигнатура которых равна 1 , а первые классы Понтрягина различны. Подсчитаем по формуле Хирцебруха второй класс Понтрягина через первый и сигнатуру: замечательно, но в ряде случаев получится дробь со знаменателем $1 / 7$, хотя числа Понтрягина гладкого замкнутого многообразия всегда целые. Итак, - это не может быть обычной 7-сферой. Милнор построил на этом многообразии функцию с двумя критическими точками (минимум и максимум), откуда следует, что оно гомеоморфно (даже кусочно-линейно) обычной сфере. Но не гладко! Замкнутые 8-мерные многообразия, полученные здесь, не гомеоморфны никакому гладкому многообразию. Правда, полное доказательство этого утверждения следует из топологической инвариантности интегралов от классов Понтрягина на циклах, установленной позднее - в 1965 году (Новиковым), но уже в 1960 году Кервер указал 10-мерное кусочно-линейное многообразие, гомотопически неэквивалентное гладкому, с помощью открытого им Arf-инварианта. Тем самым, было понято после открытия Милнора, что не все многообразия допускают гладкую структуру, и она неединственна. Том, Рохлин и Шварц доказали кусочно-линейную инвариантность интегралов от классов Понтрягина по циклам (1957), опираясь на формулу Хирцебруха и кусочно-линейные аналоги трансверсальности. Милнор и Кервер развили классификационную теорию многообразий гомотопического типа сферы с точностью до $h$-кобордизма, где многообразия эквивалентны (т.е. $h$-кобордантны), если они совместно ограничивают многообразие с двумя краями, стягиваемое к каждому из них. Они свели эту проблему к теории гомотопических групп сфер, используя метод Понтрягина. К тому времени Смейл доказал, что всякий односвязный $h$-кобордизм размерности 6 и более (с границей размерности 5 и более) тривиален, а края диффеоморфны друг другу. Он же (и независимо также Столлингс и Уоллес) доказал обобщенную гипотезу Пуанкаре, что всякое многообразие гомотопического типа сферы размерности 5 и более кусочно-линейно гомеоморфно сфере. Смейл построил функцию Морса на любом односвязном многообразии размерности 6 и более, число критических точек которой точно равно минимально 
возможному "числу Морса-Смейла", выраженному через ранги групп гомологий. Все они невырожденны. Смейлу и Хиршу принадлежит также основанное на очень красивой идее Смейла полное решение задачи о классификации гладких иммерсий (погружений с самопересечением), весьма нетривиальной уже для двумерной сферы в трехмерном пространстве, сводя ее к вычислениям гомотопических групп. Глубокие результаты были получены Хёфлигером, посвященные теории гладких вложений многообразий в многомерное евклидово пространство. Еще до работ Смейла, Марков-младший ок. 1958 г., опираясь на результаты П. Новикова, Адяна и Рабина в алгоритмической теории конечно определенных групп, установил алгоритмическую нераспознаваемость свойства многообразия размерности 4 и более быть гомотопически эквивалентным, гомеоморфным или диффеоморфным некоторому односвязному многообразию (с большим вторым числом Бетти). Любопытно, что в процессе доказательства здесь приходится установить важный частный случай леммы Смейла о "съедании ручек", однако это не было в то время осознано топологами. Полного доказательства своих теорем Марков не публиковал. Позднее С. Новиков, опираясь на леммы Смейла и указанную им конструкцию "универсального центрального расширения групп", убивающего вторую группу гомологий группы, установил алгоритмическую нераспознаваемость свойства быть $n$-сферой в размерностях 5 и более. Это - результат 1962 г., который был опубликован лишь в 70-х гг.

В начале 60-х гг. была построена "теория Браудера-Новикова", позволяющая классифицировать односвязные многообразия размерности 5 и более, гомотопически эквивалентные данному, с точностью до диффеоморфизма (С. Новиков, 1961) и выделить гомотопические типы замкнутых многообразий среди всех односвязных комплексов (В. Браудер, 1962). Эти независимые работы, решающие разные задачи, оказались чрезвычайно близки по методу, который и стал общепринятым. Фундаментальную роль в нем играет топологический аналог бирациональных отображений отображения степени 1 замкнутых многообразий, обладающие замечательными алгебро-топологическими свойствами. Классификация многообразий сводится к вычислению одной гомотопической группы "пространства Тома нормального расслоения". Поэтому теория Браудера-Новикова тесно связана с идеями теории кобордизмов. Из теории Браудера-Новикова следует, в частности, 
что имеется всего конечное (легко оцениваемое сверху) число односвязных многообразий общего гомотопического типа и с одинаковыми интегралами от классов Понтрягина по циклам (теорема Новикова). Эта теория легко переносится и на классификацию кусочно-линейных многообразий, и на многообразия с краем. Распространение этой теории на все неодносвязные многообразия размерности 5 и более было осуществлено для ряда специальных задач и частных случаев Браудером, Новиковым, Шейнсоном, и в общем случае - Уоллом около 1970 г. На теорию вложений эти методы распространил Левин в тот же период. Неодносвязные многообразия приводят к ряду интереснейших алгебраических феноменов, связанных с так называемой “алгебраической $K$-теорией" и ее эрмитовым аналогом, где еще в 1970 г. Новиков указал на значение симплектических идей. Для конечных фундаментальных групп активно привлекались в 70-х гг. методы алгебраической теории чисел. Для бесконечных групп привлекались методы функционального анализа, начиная с работ Люстига, Мищенко и Каспарова 70-х-80-х годов. Немало топологов внесло вклад в развитие и применение этих методов.

Развитие алгебраических и других методов топологии для вычисления гомотопических групп после 1956 г. также было чрезвычайно интенсивным. Э. Браун доказал алгоритмическую вычислимость систем Постникова односвязных комплексов. Алгоритмическая распознаваемость гомотопического типа среди конечных односвязных комплексов - это недавний результат Вайнбергера и Набутовского (1998). Они используют теоремы Вилкерсона и Сулливана об арифметическом характере групп гомотопических автоморфизмов конечных симплициальных комплексов $(1975 / 76)$ и глубокие результы теории алгебраических групп 1980 г.

Как указал Милнор (1957), алгебры Стинрода являются алгебрами Хопфа. С этого начинается нетривиальная теория алгебр Хопфа в топологии - ранее они рассматривались лишь как аксиоматизация колец когомологий групп Ли и пространств с умножением и единицей (как пространство петель) - т.е. $H$-пространств. Ботт, исходя из вычислений индексов Морса геодезических на группах Ли и симметрических пространствах, заметил в 1958 г. замечательное явление периодичности: до большой размерности пространство путей на унитарной группе с началом в точке 1 и концом в точке -1 гомотопически эквивалентно комплексно- 
му грассманову многообразию (о котором известно, что его пространство петель в свою очередь гомотопически эквивалентно унитарной группе до большой размерности). Тем самым, для “стабильных" гомотопических групп унитарных групп $\mathrm{U}_{n}$ (т.е. групп размерности $<2 n$, не зависящих от $n$ ), имеет место замечательный феномен 2-периодичности: эти группы зависят лишь от размерности modulo 2. Вычисления приводят к 8-периодичности для стабильных гомотопических групп ортогональных групп $\mathrm{O}_{n}$ (т.е. в размерностях меньших, чем $n-1)$. Открытие "периодичности Ботта" позволяет сделать эффективными большинство вычислений, связанных со стабильными векторными расслоениями. Из периодичности Ботта несложно извлекается теорема о несуществовании вещественных алгебр с делением во всех размерностях $n=2^{q}, q>3$, которая уже упоминалась выше как одна из знаменитых проблем алгебры, сводящаяся к топологии (Милнор, Кервер, Атья, Хирцебрух, Ботт, 1959). Мы, однако, укажем на другое решение этой же задачи (Адамс, 1959), основанное на иных методах. После создания метода Серра и др. вычисления гомотопических групп ряд топологов начал их последовательно применять и совершенствовать. Одним из первых это начали развивать Адамс, Тода, Маховальд и др., получившие много ценных результатов. Адамс в 1958 г. открыл замечательный метод изучения стабильных гомотопических групп (для $(n-1)$-связных пространств это - гомотопические группы размерности меньшей, чем $2 n-1)$. Для $n$-сфер стабильная гомотопическая группа с номером $k$ зависит лишь от разности размерностей $(k-n)$ и нетривиальна для $k-n \geqslant 0$. Прямая сумма всех стабильных групп сфер это суперкоммутативное градуированное кольцо относительно суперпозиции отображений сфер в сферы. Для вычисления стабильных гомотопических групп была открыта "спектральная последовательность Адамса", второй член которой выражается через гомологическую алгебру для когомологий как модулей над алгеброй Стинрода. Адамс решил проблему инвариантов Хопфа (и тем самым проблему алгебр с делением) исходя из этого метода для $p=2$. Научная юность автора этих строк лежит именно здесь, в 1958-59 гг., развивая этот метод для $p>2$, чтобы найти "длинные суперпозиции" сфер, усовершенствуя вычисления с алгебрами Хопфа, вводя для этого своеобразные аналоги операций Стинрода в гомологии алгебр Хопфа над конечным полем. Милнор и Новиков в 1960 г. применили метод Адамса к задаче вычис- 
ления колец ориентированных кобордизмов; ими же введены унитарный (комплексный) аналог кобордизмов, специальный унитарный, спинорный и симплектический. Милнор, как свидетельствуют западные топологи, пришел к этим идеям несколько раньше, но впервые написал и опубликовал лишь часть в 1960 г., содержащую доказательство теоремы об отсутствии нечетного кручения в ориентируемых кобордизмах и отсутствии кручения в комплексных кобордизмах, методом Адамса. Свою оригинальную геометрическую идею доказательства полиномиальности этих колец, сообщенную им лично в 1961 г., Милнор отложил до второй части, которая так и не была написана. Много позднее другие западные авторы опубликовали доказательство, реализуя идею Милнора (но не упоминая его). Новиков, формализуя хопфовские свойства некоторых модулей когомологий над алгеброй Стинрода как "коалгебр", использовал мультипликативные свойства спектральной последовательности Адамса для универсальных пространств Тома и вычислил кольцевую структуру как элементарное следствие алгебраических аргументов. Ряд результатов был получен им о кольцах специальных унитарных, а также симплектических кобордизмов (1962). Последние не вычислены и сейчас, хотя второй член спектральной последовательности Адамса здесь найден Ивановским и Вершининым в 1970-х гг. Ряд результатов получен Ботвинником и Кочманом в 1970-80-х гг., но проблема очень сложна. Спинорные кобордизмы проще и были вычислены Брауном и Петерсоном (1965); они успешно применяли кобордизмы, решив известную проблему Arf-инварианта в теории гладких структур на сферах для размерностей $8 k+2$. Наиболее глубокий результат здесь получен Браудером (1970), но проблема все еще открыта в размерностях $2^{k}-2$ при $k>5$. Для изучения 2-кручения в ориентируемых кобордизмах геометрический метод был развит Рохлиным (1958), но он допустил ошибку. Окончательный результат был получен Уоллом (1960). Новиков доказал также, что проблема Стинрода разрешима в ориентируемом случае для любого $k$-мерного цикла, умноженного на некоторое нечетное число. Это число может быть взято равным 1 , если в некоторых гомологиях нет специального нечетного кручения в ориентируемом случае (и аналогично в унитарном, без условия нечетности).

Развитие алгебраических методов топологии и теории гомологий в этот период приобрело чрезвычайно любопытные чер- 
ты, особенно после работы Гротендика, нашедшего совершенно новое понимание теорем типа Римана-Роха, фундаментальных в алгебраической геометрии. В частности, он ввел так называемый " $K$-функтор", перенесенный Атьей и Хирцебрухом в топологию. В частности, вместо четномерных коциклов берутся стабильные классы унитарных расслоений в чисто топологическом варианте. Эти группы (кольца) $\mathbb{Z}_{2}$-градуированы, они удовлетворяют всем аксиомам Эйленберга-Стинрода, кроме "нормировки": $K$-функтор точки нетривиален и представляет собой кольцо лорановских полиномов от оператора периодичности Ботта (и ему обратного) над целыми числами. Подобные объекты, вроде когомологий, но без нормировки, стали называться "экстраординарными теориями когомологий". Их аксиоматика и очень общие свойства систематизировались Уайтхедом-вторым в начале 60-х гг. Конкретно, $K$-теория эффективно вычислима в силу периодичности Ботта. Роль когомологических операций в ней играют представления унитарных групп или их специальные линейные комбинации - например, внешние степени. Содержательную теорию здесь развил в первую очередь Атья, указав на возможные топологические применения. Он же указал на теорию кобордизмов как на экстраординарную теорию когомологий и гомологий и установил ряд ее важных свойств. Например, двойственность Атьи для комплексов Тома оказалась важной в классификационной теории многообразий Браудера-Новикова, сводя ее к универсальным пространствам, как указал ряд авторов в 1965/66 гг.

В знаменитой работе Атьи-Зингера (1963) $K$-теория была успешно применена к известной проблеме вычисления индекса эллиптических дифференциальных операторов, начатой Нётером и Мусхелишвили еще в 30-х гг. для сингулярных интегральных операторов на окружности, развитая рядом советских математиков. Она была сформулирована Гельфандом (1961) как общематематическая проблема и вскоре решена Атьей и Зингером. Значения этих идей для анализа трудно переоценить. Инварианты эллиптического оператора удачно формулируются в $K$-теории: от многообразия в формулу индекса входят классы Понтрягина.

Адамс в 1962 г. построил линейные комбинации внешних степеней, обладающие замечательными свойствами в $K$-теории как когомологические операции ("операции Адамса"). С помощью них Адамс решил несколько известных задач топологии: он доказал 
гипотезу Тода о том, что на $n$-сфере нельзя построить линейно независимых векторных полей больше, чем известно исходя из инфенитезимальных вращений, и дал оценку сверху важных подгрупп в стабильных гомотопических группах сфер, ранее красиво оцененных снизу Милнором через числа Бернулли исходя из тех же формул типа Хирцебруха. Нижняя и верхняя оценка в конечном счете совпали для этих специальных подгрупп. Полные доказательства были опубликованы лишь несколько лет спустя (1965-66). Он сформулировал "гипотезу Адамса" о порядках конечных абелевых групп Дольда классов послойно гомотопически эквивалентных стабильных сферических расслоений, важных для топологии, а также поставил задачу нахождения аналога спектральной последовательности Адамса для вычисления стабильных гомотопических групп в $K$-теории вместо обычных когомологий. Последняя задача оказалась неразрешимой в рамках $K$-теории. Новиков (1966-67) реализовал эту программу, заменив $K$-теорию на комплексные кобордизмы. Уже раньше ориентируемые и унитарные (комплексные) кобордизмы были эффективно использованы для исследования неподвижных точек гладких преобразований конечного порядка и для завершения вычислений специальных унитарных кобордизмов (Коннер и Флойд, 1964/65). Они же ввели аналоги классов Черна векторных расслоений, принимающие значения в унитарных (комплексных) кобордизмах вместо обычных гомологий. Это крайне естественно - еще в начале 50-х гг. Гамкрелидзе изучал циклы Черна как линейные комбинации алгебраических подмногообразий. Напомним, что именно в кобордизмах циклы представляются отображениями многообразий тех или иных типов. Новиков и Ландвебер ввели аналоги степеней Стинрода в комплексных кобордизмах (1966). Порожденная ими алгебра является алгеброй Хопфа над целыми числами и носит название алгебры Ландвебера-Новикова. Роль скаляров в теории кобордизмов как теории когомологий (или когомологий точки) играет кольцо комплексных кобордизмов Милнора-Новикова. Согласно теореме Новикова, все когомологические операции этой теории получаются применением алгебры Ландвебера-Новикова и затем домножением на кольцо "скаляров" - выше. Однако эти две алгебры не коммутируют. Их коммутационные соотношения (формулы Новикова) завершают описание аналога алгебры Стинрода этой теории. Как заметил позднее Бухштабер (1978), это - две дуальные алгебры Хопфа 
с любопытной целочисленной структурой. Новиков и Бухштабер уже в 90-х гг. изучали возникшую отсюда общую конструкцию в теории алгебр Хопфа, назвав ее "операторным дублем"; Л. Фаддеев и Семенов-Тян-Шанский, пришедшие к этому тогда же из других идей, назвали это "гайзенбергов дубль". Построение "спектральной последовательности Адамса-Новикова" для вычисления стабильных гомотопических групп сфер и других пространств принесло немало результатов. Ряд топологов развивали эти идеи. Особо отметим введенную в 1967 г. Новиковым и Мищенко формальную группу геометрических кобордизмов для вычисления аналога первого класса Черна тензорного произведения, комплексных расслоений ранга 1; был найден ряд ее применений, например, для построения аналогов операторов Адамса и характера Черна (Новиков), и позднее - характера ЧернаДольда (Бухштабер). Квиллен в 1969 г. идентифицировал эту формальную группу с универсальной формальной группой Лазара в чистой алгебре и успешно применил ее теорию для вычисления ряда топологических величин - в частности, для эффективного вычисления проектора из теории кобордизмов на ее прямое слагаемое (теория Брауна-Петерсона) над кольцом целых $p$-адических чисел, чрезвычайно полезного для вычисления стабильных гомотопических групп сфер методом Адамса-Новикова. Развитие идей теории формальных групп привело Новикова и Бухштабера к понятию двузначной формальной группы (1971). Теория многозначных групп была далеко развита Бухштабером в 70-х гг. Новиков, Бухштабер, Гусейн-Заде и Кричевер в конце 60-х-начале 70-х гг. успешно применяли эти методы для вычисления инвариантов неподвижных точек гладких действий циклических групп и окружности на многообразиях. Значительно позднее, в конце 80-х гг., замечательные топологические соотношения для действий окружности нашли квантово-полевые физики, в частности Виттен. Здесь приняли участие также топологи Ошанин и Ландвебер, соединившие новые идеи с классической теорией комплексных кобордизмов начала 70-х гг. Выявилась особая роль так называемых эллиптических родов от характеристических классов, обладающих "свойством жесткости". В последнее десятилетие на том же пути было вскрыто немало любопытных соотношений между характеристическими числами Понтрягина и Черна, особенно для специальных унитарных кобордизмов (их стали называть в новой теории "многообразиями Калаби-Яо"). 
В этих соотношениях проявляется только природа теории кобордизмов, а отнюдь не метрики типа Калаби-Яо. Возможности теории кобордизмов еще далеко не исчерпаны. Естественно ожидать, что и в теории особенностей, развитой Арнольдом и его школой, кобордизмы должны сыграть активную роль вместо теории обычных гомологий.

Непрерывные гомеоморфизмы в топологии. Особую роль в топологии играет проблема непрерывных гомеоморфизмов. В отличие от диффеоморфизмов, устойчивых среди всех гладких отображений в соответствующей топологии или гомотопических эквивалентностей, непрерывные гомоморфизмы неустойчивы среди всех непрерывных отображений: взаимная однозначность исчезает при случайном малом возмущении, если производные не контролируются (или не существуют). Это делает проблему гомеоморфизма особенно трудной. В конечном счете оказалось, что для гладких замкнутых односвязных многообразий гомеоморфность эквивалентна гомотопической эквивалентности в размерностях 2, 4, 5 и, вероятно, 3 (гипотеза Пуанкаре), для $n=4$ это - теорема Фридмана (1984), а для $n=5$, как установил Новиков в 1964, они даже диффеоморфны. Однако при $n>5$ картина меняется: основными величинами отличающими гомеоморфизм от гомотопического типа являются интегралы от классов Понтрягина по циклам, топологическую инвариантность которых установил Новиков (1965) - см. ниже.

Топология XX века долго исходила из предположения, что все ее существенные законы инвариантны относительно непрерывных гомеоморфизмов, хотя определение важных величин всегда производилось, используя дополнительные структуры: комбинаторная техника (разбиение на симплексы и превращение в симплициальный комплекс) начиная с Пуанкаре была основой строгого определения гомологий и фундаментальной группы, позднее - кручения Радемайстера-Уайтхеда. Использование гладкой структуры стало возможно после открытий Уитни 30-х гг: характеристические классы Штифеля-Уитни и Понтрягина были введены как инварианты гладкой структуры - либо через интегралы по циклам определенных выражений от римановой метрики, либо через циклы особенностей векторных и тензорных полей, либо через аналог гауссова отображения. С самого начала существования топологии как точной науки, построенной на комбинаторной основе, возникла "Hauptvermutung" - основная гипотеза 
комбинаторной топологии, - утверждающая, что непрерывно гомеоморфные симплициальные комплексы (в частности, кусочнолинейные многообразия) на самом деле комбинаторно (кусочнолинейно) эквивалентны. Подобная гипотеза предполагалась верной и для гладких непрерывно гомеоморфных многообразий (что они на самом деле диффеоморфны), пока ее не опроверг Милнор в 1956 г. - см. выше. Фактически Милнор вскрыл различие между гладкими и кусочно-линейными структурами: бывают различные гладкие многообразия, кусочно-линейно изоморфные друг другу, но не диффеоморфные - в размерностях 7 и выше. Бывают кусочно-линейные многообразия размерности 8 и выше, не допускающие гладкой структуры: некоторые из таких многообразий можно задать одним комплексным уравнением в проективном пространстве с одной изолированной особенностью. Созданная рядом авторов в 1958-65 гг. теория препятствий, различающих гладкие и кусочно-линейные структуры (Том, Манкрес, Хирш и др.), лежит в когомологиях со значением в конечных абелевых группах диффеоморфизмов сферы по модулю продолжаемых на диск, совпадающих с группами гладких структур на сферах, найденных Милнором и Кервером во всех размерностях, кроме 4. Недостающая здесь группа препятствий на 4-остове была найдена Серфом в 1966 г. Она равна нулю. То есть, по модулю конечных чисел эти две категории - гладкая и кусочно-линейная - для многообразий размерностей 5 и выше эквивалентны. Топологические гипотезы оказались "почти правильны" в этом случае. Даже некоторые из кручений классов Понтрягина оказываются кусочно-линейно неинвариантными для многомерных многообразий в целочисленных когомологиях (например, 7-кручение второго класса), хотя интегралы от них по циклам комбинаторно инвариантны, как уже указано выше. Теория кусочно-линейных многообразий оказывается очень похожей на гладкую теорию по методам и результатам, с соответствующим использованием аналога идей трансверсальности, аналогов векторных расслоений и т.д. Классификационная теория Браудера-Новикова также легко переносится на кусочно-линейные многообразия, заменив векторные расслоения на так называемые кусочно-линейные микрорасслоения Милнора.

Как быть с чисто непрерывными гомеоморфизмами? В малых размерностях любой непрерывный гомеоморфизм аппроксимируется кусочно-линейным и даже диффеоморфизмом. Для 
трехмерных многообразий это доказал Мойс в начале 50-х гг., и это - наиболее сильный результат, который можно здесь получить прямыми элементарными методами. Около 1960 г. М. Браун и Мазур доказали многомерный аналог трехмерной теоремы Шенфлиса: всякое вложение $(n-1)$-сферы в $n$-мерное евклидово пространство ограничивает $n$-мерный диск, если окрестность сферы "ручная", т.е. имеет вид прямого произведения на интервал (бывают уже в трехмерном случае "дикие" вложения сфер Александера, где это не так). Мазур красиво и элементарно доказал также, что два замкнутых многообразия одинакового гомотопического типа с учетом типа касательного векторного расслоения становятся диффеоморфными друг другу после умножения на евклидово пространство большой размерности (1960) - хотя, конечно, сами они могут быть недиффеоморфными друг другу. Различия стираются после умножения на многомерное открытое евклидово пространство. Как показал Милнор, используя эти аргументы для линзовых многообразий, отсюда следует опровержение Hauptvermutung: если гомотопически эквивалентные линзовые многообразия (которые всегда параллелизуемы в трехмерном случае) имеют различные детерминанты Радемайстера, то пространства Тома эквивалентных расслоений над ними комбинаторно неэквивалентны, но гомеоморфны. Например, для тривиальных расслоений комплексы Тома получаются из прямых произведений линз на $n$-мерный диск стягиванием всей границы в точку. Это - особая точка. Версия инварианта Радемайстера "по модулю особой точки" является комбинаторным инвариантом, который совпадает с инвариантом Радемайстера линз; поэтому различны комбинаторные классы этих гомеоморфных комплексов. Эти аргументы приводят к примерам комплексов в размерности 6 и более. Далее, Милнор высказал гипотезу, что двукратная надстройка над любым трехмерным многообразием - гомологической сферой - всегда гомеоморфна 5-сфере. Эта гипотеза была доказана 15 лет спустя Эдвардсом в 70-х гг. путем прямого построения гомеоморфизма. Его геометрический метод опирается на конструкции "разрешения диких вложений", развитые Штанько. Разумеется, это показывает, что на 5-сфере имеются чрезвычайно сложные триангуляции, где она даже не является кусочнолинейным многообразием. Разумеется, они комбинаторно неэквивалентны обычной 5-сфере со стандартной триангуляцией. 
Как же все-таки обстоит дело с важнейшими инвариантами гладких и кусочно-линейных многообразий? Все обсуждавшиеся контрпримеры не являются кусочно-линейными многообразиями. Как уже говорилось, гомологии и гомотопические группы гомотопически инвариантны. Классы Штифеля-Уитни также являются гомотопическими инвариантами замкнутых многообразий, они не способны различать гомеоморфизм и гомотопический тип. Интегралы от классов Понтрягина по циклам не являются гомотопическими инвариантами, вообще говоря, как указывалось выше.

В 1964/65 гг. Новиков нашел совершенно новый подход к изучению непрерывных гомеоморфизмов, позволивший ему доказать топологическую инвариантность интегралов от классов Понтрягина по циклам, т.е. их сохранение при чисто непрерывных гомеоморфизмах гладких и кусочно-линейных многообразий. В основе этого подхода лежит локализация интегралов ПонтрягинаХирцебруха в специальных "торических" областях на многообразии с последующим развитием и использованием далеко идущей техники дифференциальной и алгебраической топологии для изучения многообразий торического типа со свободной абелевой фундаментальной группой. Например, в частном случае нужно доказать, что классы Понтрягина многообразий гомотопического типа тора равны нулю.

Этот подход имеет идейное родство с так называемой "эталь-топологией" Гротендика, где была использована в конце 50-х-начале 60-х гг. категория накрытий над открытыми областями в топологии Зариского, организованная в “этальтопологию", чтобы правильно ввести гомологии алгебраических многообразий через их реализацию над полями конечной характеристики. Все дальнейшие глубокие результаты топологии непрерывных гомеоморфизмов используют этот подход или его дальнейшее развитие. Из инвариантности классов Понтрягина следует, что на односвязном замкнутом многообразии размерности 5 и более имеется лишь конечное число гладких или кусочно-линейных структур. Развитие этого метода позволило также установить верность Hauptvermutung для односвязных многообразий размерности 5 и более, если в 3-мерных гомологиях нет 2-кручения - Сулливан, 1967. Любопытно, что последнее ограничение на 3 -мерные гомологии было им сначала не замечено и указано ему Новиковым и Браудером. Без сомнения, это свидетельствует о весьма сыром состоянии, в котором тогда находились 
эти результаты. Следует заметить, что теория Сулливана в полном объеме до сих пор строго не написана, хотя ряд ее компонент был доказан и опубликован позднее другими авторами: необходимые результаты из теории гомотопий были получены Мадсеном и Мильгрэмом в 80-х гг. с использованием других идей, ряд других компонент может быть обоснован исходя из техники, развитой Квином в 80-х гг. Однако единого полностью написанного доказательного изложения теории Сулливана пока нет в литературе. Более слабые теоремы были доказаны проще и в полном объеме: Hauptvermutung для 3-связных многообразий (ЛашофРотенберг, 1968) и даже для односвязных многообразий, у которых группа 3-гомологий по модулю 2 тривиальна (Кассон, 1969, опубликовано много позднее). Все эти результаты о непрерывных гомеоморфизмах опираются на торическую конструкцию, введенную Новиковым. В 1968 Кирби предложил модификацию торической конструкции и красиво применил ее к известной Проблеме Кольца: доказать, что всякая область в евклидовом пространстве, ограниченная парой "ручных" сфер, гомеоморфна цилиндру (т.е. произведению $(n-1)$-сферы на замкнутый отрезок). Для гладкого случая размерности $n=6$ и выше это следует из теоремы Смейла, но чисто непрерывный случай оказался труден. Метод Кирби сводит эту проблему к теории гладких структур на многообразиях гомотопического типа $n$-тора, но от гомотопических торов требуется больше, чем в теории классов Понтрягина: надо доказать не только то, что они все параллелизуемы, но и то, что различия между ними стираются при переходе к достаточно большому конечнолистному накрытию. Доказательство этого было завершено Зибенманом для всех размерностей 5 и более. Метод Кирби привел к ряду сильных результатов: опираясь на теорему Чернавского о локальной стягиваемости группы непрерывных гомеоморфизмов многообразий, Кирби и Зибенман в конце 60-х гг. показали, используя всю совокупность методов топологии, что Hauptvermutung неверна для кусочно-линейных многообразий высокой размерности. В их работе инвариант, различающий два многообразия, принимает значения в 2-кручениях 3-гомологий. Он происходит из так называемой "рохлинской двойки" - различии между 12 и 24 при вычислении порядка $(n+3)$-й стабильной гомотопической группы $n$-сфер, где Рохлин сделал упоминавшуюся выше ошибку, имеющую глубокую природу. Опираясь на результаты Сяна и Фаррела (1968), Кирби и Зибенман 
построили в 70-х гг. классификационную теорию непрерывных многообразий. Однако последние работы, в отличие от Проблемы Кольца и опровержения Hauptvermutung, опираются на уже упоминавшуюся теорию Сулливана, так что их полное доказательство тем самым тоже пока не содержится в литературе.

Интересная идея была предложена Сулливаном в середине 70-х гг: он предложил конструкцию липшицевых структур на многообразиях и набросок доказательства их существования и единственности в чисто непрерывных многообразиях (использующую решение Проблемы Кольца, так что этот факт никак нельзя считать элементарным). Однако доказательства этих теорем никогда и нигде не были полностью написаны. Позднее Сулливан совместно с Телеманом, а также Вайнбергер в 90-х гг. начали построение анализа и теории операторов на липшицевых многообразиях. Как побочный продукт теории, приведшей к доказательству топологической инвариантности классов Понтрягина, Новиков высказал гипотезу классификации гомотопически инвариантных выражений от классов Понтрягина (1970): это - интегралы от полиномов Понтрягина-Хирцебруха по циклам, двойственным к классам когомологий, сидящим на фундаментальной группе (гипотеза Новикова или гипотеза высших сигнатур). Начиная с работы Люстига (1971), целый ряд авторов получил здесь глубокие результаты методами геометрии и функционального анализа. Среди них - А. Мищенко, Каспаров, Громов, А. Кон и ряд других. Эта гипотеза в полном объеме до сих пор не доказана. В 1974 г. Чепмен доказал топологическую инвариантность кручения Радемайстера-Уайтхеда красивым элементарногеометрическим методом. Чрезвычайно интересные свойства были открыты в 80-х и 90-х гг. Лаусоном и Громовым для односвязных многообразий положительной скалярной кривизны: свойство допускать такую метрику является инвариантом спинорных кобордизмов. В неодносвязном случае имеется аналог гипотезы Новикова (гипотеза Лаусона-Громова-Розенберга), где полиномы Хирцебруха заменены на так называемый $A$-род, возникающий в теории оператора Дирака. Теорию кобордизмов в проблемах геометрии успешно использовали Крек и Штольц в 90-х гг.

Возвращаясь к проблемам алгебраической топологии и теории гомотопий, следует обратить внимание на красивую общекатегорную конструкцию " $p$-адической локализации" гомотопических типов (не только стабильных, но и всех односвязных комплексов и 
даже несколько более), которая была предложена Сулливаном и Квилленом ок. 1970 г. и позволила доказать уже упоминавшуюся гипотезу Адамса (выше) об оценке порядка важной для классификационных проблем топологии группы послойных гомотопических классов стабильных векторных расслоений, - через операторы Адамса в $K$-теории. В этой конструкции решающую роль играет то, что грассмановы многообразия алгебраически определены над целыми числами, так что общекатегорный характер этого метода, позволившего решить трудную в конкретных примерах проблему, является очень любопытным. В последующие годы был получен ряд ценных результатов в теории гомотопий: открыты в 70-х гг. нетривиальные конечномерные $H$-пространства, не сводящиеся к группам Ли и 7-сфере (Мислин, Хилтон), установлена нильпотентность любого элемента в кольце стабильных гомотопических групп сфер (Нишида), произведен ряд очень ценных построений и вычислений на базе методов теории кобордизмов рядом авторов, включая Вильсона, Равенела, Бухштабера, Мораву и др. В 70-х гг. была построена полезная чисто кольцевая теория "минимальных моделей" рационального и вещественного гомотопического типа, удачно сформулированная Сулливаном исходя из кольца Уитни-Тома симплициальных дифференциальных форм на комплексах (т.е. набора форм на симплексах, совпадающих после ограничения на общую границу). Сулливан указал здесь подкольцо с рациональными коэффициентами, выделяемое функториально. Это приводит к модели рационального гомотопического типа. Она оказывается в ряде случаев эффективным средством изучения топологии конкретных многообразий - келеровых многообразий, где весь рациональный тип определяется кольцом когомологий, как показали Сулливан, Делинь, Гриффитс, Морган в совместной работе, однородных пространств и других. Интересные аналитические идеи, начатые ряд лет до этого Ченом, - как написать гомотопически инвариантные выражения на отображениях окружности (еще в 50-х гг.) и позднее сфер в 70-х гг. в многообразия через "итерированные" интегралы в кольце дифференциальных форм, - получили в рамках кольцевой теории гомотопического типа весьма естественную трактовку. Здесь имеются интересные алгебраические конструкции и проблемы, связанные с проблемой эффективного выделения рациональных интегралов исходя из аналитически заданного кольца гладких дифференциальных форм, начатой Новиковым в 1984 г. для нужд теории 
поля. Напомним, что впервые интегралы такого рода на гомотопических группах сфер написал для инварианта Хопфа Уайтхед в 50-х гг., исходя из интеграла Кельвина XIX века в вихревой гидродинамике.

Подводя итоги периода наивысшего расцвета классической алгебраической топологии (т.е. 50-60 гг.), мы видим, что и позднее, в 70-80 гг., в этой области было получено немало глубоких результатов. Однако сообщество топологов все менее и менее видело значение своих идей для остальной математики, все более и более сужало область своих интересов и свой горизонт, делало свой язык все более и более изолированным и абстрактным. Кроме того, как указывалось выше, сообщество потеряло контроль за степенью доказанности даже лучших результатов своей области. Безусловно, последняя черта - это свидетельство снижения уровня этой области, когда центральные теоремы не доказаны, а следующие поколения даже этого не знают и "верят классикам".

Следует сказать, что классическая алгебраическая топология периода 1950-1970 годов, за исключением отдельных кусков, не описана ни в каком цикле общедоступных учебников: имеющиеся пособия либо затрагивают лишь ее начальный этап, либо немыслимо абстрактны. Ее лучше всего изучать по основным работам, которые в тот период были ясно и подробно написаны (их список можно найти в [1], [2]). В конце приложен список книг, более удобных для чтения, чем подавляющее большинство других.

Как будет видно ниже, в период 1985-1995 гг. топология пережила новый период подъема, основанного на совершенно других идеях, где ведущую роль сыграли идеи (а зачастую и люди), пришедшие из мира квантовой теории.

\section{Маломерная и гиперболическая топология}

Маломерная топология 1950-х гг. началась с доказательства так называемой "леммы Дена" Папакирьякопулосом в 1957 г.: первая попытка ее доказать была сделана еще Деном в начале XX века, но обнаружился глубокий пробел. Эта теорема утверждает, что узел в трехмерном пространстве тривиален, если фундаментальная группа дополнительного пространства абелева (циклическая). Была доказана также "теорема о сфере": если вторая группа гомотопий трехмерного многообразия отлична от нуля, то найдется вложенная гомотопически нетривиальная 2-сфера. Милнор 
указал ряд красивых следствий этого результата: все дополнительные пространства узлов в 3-сфере асферичны. Любое замкнутое трехмерное многообразие представляется в виде связной суммы "элементарных". Среди ориентируемых 3-многообразий элементарные многообразия - это прямое произведение 2-сферы на окружность, многообразия с конечной фундаментальной группой и многообразия, у которых универсальная накрывающая стягиваема. В течение 60-х гг. Хакен и Вальдхаузен построили глубокую структурную теорию 3-многообразий и специальных "неприводимых" поверхностей в них. Доказано, что топологический тип узла вполне определяется фундаментальной группой с отмеченной абелевой подгруппой (Вальдхаузен, конец 60-х гг.). Хакен построил алгоритм, распознающий свойство узла быть тривиальным, хотя этот алгоритм долго не удавалось реализовать практически. Начала развиваться программа исследований, имеющая своей целью доказать алгоритмическую разрешимость свойства пары узлов (зацеплений) быть изоморфными. Ряд последователей Хакена участвовал в этом, но до последнего времени оставались пробелы. Возможно, в начале XXI века Матвееву удалось завершить эту программу, используя свою теорию сложности 3-многообразий, но его работа пока не проверена. В 90-х гг. Рубинштейн и Томпсон построили алгоритм, распознающий 3-сферу методами теории Хакена.

В конце 70-х гг. М. Фридману удалось полностью завершить программу, идея которой была первоначально выдвинута Кассоном, но оказалась весьма трудной: используя модификации многомерных аргументов типа Смейла и производя их бесконечными сходящимися сериями, оказалось возможным доказать, что односвязные гомотопически эквивалентные замкнутые 4-многообразия непрерывно гомеоморфны - в частности, доказан чисто непрерывный аналог гипотезы Пуанкаре для 4-сферы. Позднее мы вернемся к проблеме диффеоморфизма 4-многообразий. Пока удается только устанавливать негативные теоремы, что многообразия не диффеоморфны друг другу (ниже). Прогресса в положительном направлении не видно.

Среди результатов, примыкающих к топологии, широкой известностью пользуется теорема Аппеля-Хакена конца 80-х гг, устанавливающая верность классической гипотезы о возможности раскраски конечной плоской карты четырьмя красками. Необычным в этой работе является грандиозное по масштабу тех лет 
применение компьютерных расчетов в самой математике. $\mathrm{K}$ настоящему времени этот результат считается проверенным.

В 70-х гг. Тёрстон открыл, что на очень многих трехмерных многообразиях существует метрика постоянной отрицательной кривизны. Это относится, в частности, ко многим дополнительным пространствам узлов, которые получаются из дискретных групп движений трехмерного пространства Лобачевского ("гиперболического пространства"), где фундаментальная область имеет конечный объем. Тёрстон разработал глубокие методы изучения и построения гиперболических многообразий. Он выдвинул "гипотезу геометризации", утверждающую, грубо говоря, что если нет самоочевидных препятствий, то 3-многообразие допускает метрику постоянной отрицательной кривизны. Для замкнутых многообразий это означает, что фундаментальная группа бесконечна, неразложима в свободное произведение и все ее абелевы подгруппы циклические; опасаясь опровержения гипотезы Пуанкаре, мы скажем, что в этом случае многообразие должно быть гомотопически эквивалентно многообразию отрицательной кривизны, но гипотеза Тёрстона является гораздо более общей. Реализация идей Тёрстона для специального класса "многообразий Хакена" была завершена Оталом, который дал полное доказательство в этом случае в 90-х гг.; гипотеза доказана и в некоторых других частных случаях. Кстати, дополнительные пространства узлов - это многообразия Хакена. Немало специалистов развило эти идеи. Любопытная алгебра Ли была открыта Голдманом для гомотопических классов замкнутых путей на поверхностях, - как побочный продукт исследований, связанных с пространствами модулей римановых поверхностей. Исследования топологии пространств модулей римановых поверхностей и векторных расслоений - это глубокая актуальная тема, не входящая в круг идей, который мы здесь обсуждаем. Возвращаясь к теории узлов и зацеплений, мы отметили здесь, что в самое последнее время, уже в XXI веке, Дынников развил совсем новый алгебраический подход к проблеме классификации узлов и зацеплений через представление их в "многостраничной книге", составленной из $k$ листов (полупространств, склеенных вдоль “общей оси"), где число $k$ не менее трех. Такое представление вводилось еще 100 лет назад, но Дынников обнаружил его замечательные алгебраические свойства: проблема классификации узлов и зацеплений сводится к вычислению центра конечно определенной полугруппы 
с единицей. В рамках теории групп ничего подобного не удается сделать. Алгоритм распознавания тривиального узла получается здесь немыслимо простым и доступным, ряд следствий вытекает и для групп кос. По-видимому, это - чрезвычайно удачный подход.

Недавно Перельман начал представлять последовательность текстов, ведущих (как утверждается) к доказательству гипотезы Пуанкаре: аналитическим методом "потока Риччи" с некоторыми исправлениями на гомотопической трехмерной сфере вводится метрика положительной кривизны. Ряд крупнейших экспертов подтверждает, что в этих текстах имеются чрезвычайно интересные перспективные идеи. В данный момент мы не знаем, приводят ли эти идеи к желаемому результату.

Следует отметить, что в 80-х и начале 90-х имело место далеко идущее идейное развитие в теории узлов и трехмерных многообразий; были открыты замечательные полиномиальные инварианты узлов - такие, как полином Джонса и его обобщения (полином HOMFLY) около 1984/85 гг., по-новому осмыслен классический полином Александера. Вскоре стало ясно, что полиномы типа Джонса более естественно строятся, как указал Тураев, в рамках модели Александера-Маркова через своеобразные серии представлений групп кос: они строились исходя из частных случаев решений так называемых "уравнений Янга-Бакстера", использовавшихся для точного решения моделей статистической физики и квантовой теории поля; оказалось, что это - представления групп кос в нужных случаях. Геометрические свойства полинома Джонса позволили доказать гипотезу Тейта, сформулированную в конце XIX века: исключая тривиальные случаи, число точек пересечения в альтернированной диаграмме узла является топологическим инвариантом (Кауфманн и Мурасуги, 1987). Это не удавалось доказать методами классической алгебраической топологии. В конце 80-х гг. большое внимание топологов привлекла теория так называемых "инвариантов Васильева" конечного порядка. Их полная классификация над полем характеристики нуль дана Концевичем в конце 1992 г., метод которого использует идеи, заимствованные из квантовой теории. Над конечными полями эта проблема открыта. Свойство топологического инварианта иметь "конечный порядок" в смысле Васильева - это очень полезная общая идея. Коэффициенты полиномов Александера, Джонса и их обобщений оказываются такими, как доказано Бар-Натаном, 
Бирман и Лином в начале 90-х гг.

Виттен заметил в конце 80-х гг., что полином Джонса имеет квантово-полевую трактовку как известная в теории полей ЯнгаМиллса связанная с замкнутой кривой корреляционная функция - "петля Вильсона". В данном случае мы имеем 3-мерную теорию Янга-Миллса с весьма специальным “топологическим" функционалом действия - так называемым функционалом ЧернаСаймонса. Идея топологической квантовой теории поля имеет любопытную историю. В начале 70-х гг. Зингер и Рей начали теорию детерминантов эллиптических операторов и развили аналитический подход к инварианту Радемайстера, усовершенствованный Атьей. Значительно позднее в 80-х гг. было доказано, что аналитическое кручение Рея-Зингера действительно совпадает с комбинаторным кручением Радемайстера (Мюллер и Чигер), используя симплициальную аппроксимацию операторов Лапласа-Бельтрами на дифференциальных формах.

В конце 70-х гг. А. Шварц поставил вопрос: можно ли строить топологические инварианты многообразий как квантово-полевые функциональные интегралы по всем полям (что бы это ни означало) - таким, что действие не зависит от римановой метрики? Он рассмотрел простейшую абелеву калибровочную теорию на 1-формах в 3-многообразии, заданную действием КельвинаУайтхеда (плотность инварианта Хопфа), где калибровочное преобразование состоит в добавке к 1-форме точного слагаемого, не меняющего действие. Проводя последовательную теорию этого калибровочного поля (включая построение "духов ФаддееваПопова"), Шварц показал, что возникающей величине можно придать смысл: она совпадает с кручением Радемайстера-Рея-Зингера. Вообще, теория детерминантов стала очень популярной в калибровочно-инвариантных теориях поля в связи с “духами Фаддеева-Попова". В конце 80-х гг. Виттен начал активное развитие топологических квантовых теорий поля на различных многообразиях, особенно размерностей $2,3,4$. В трехмерном случае для простейшей неабелевой теории Янга-Миллса с функционалом Черна-Саймонса в качестве действия Виттен обнаружил, что так называемая "петля Вильсона", если ее точно определить, совпадает с полиномом Джонса узлов. Не вдаваясь глубоко в эту теорию, мы укажем на ее чисто топологическую аксиоматику Атьи-Сигала: любая такая "теория", называемая TQFT, сопоставляет любому $(n-1)$-мерному замкнутому ориентированному мно- 
гообразию конечномерное евклидово пространство, объединению многообразий - их тензорное произведение. Любому $n$-мерному ориентированному многообразию с краем она сопоставляет отмеченный вектор в евклидовом пространстве, соответствующем его границе - с учетом ее ориентации. Если граница состоит из нескольких компонент, то соответствующее ей пространство это вектор в тензорном произведении, у него есть тензорные индексы. После склейки двух многообразий с краем вдоль целой компоненты границы новый отмеченный вектор получается из тензорного произведения двух отмеченных векторов сверткой по всем индексам, отвечающим общей компоненте. В классической топологии было известно только два примера: в обоих примерах все пространства одномерны и отмеченный элемент - это экспонента или от эйлеровой характеристики многообразия с краем $(n=2 k)$, или от его сигнатуры $(n=4 k)$. Обоснование поведения при склейке дает лемма об аддитивности при склейке вдоль целой компоненты границы для эйлеровой характеристики и "лемма Новикова-Рохлина" (1965/66) об аддитивности сигнатуры при склейке, которая согласно Яниху (1967) является аксиомой, определяющей эти две величины. TQFT с пространствами размерности, больше чем 1 , возникли только из современной квантовой теории. Новые топологические инварианты, например, трехмерных многообразий, получаются из TQFT; если представить 3-многообразие склейкой двух многообразий с краем вдоль общей границы, этот инвариант равен скалярному произведению отмеченных векторов. Эффективное комбинаторное построение TQFT - это непростая задача. Она была решена в некоторых случаях Тураевым-Решетихиным и Виро-Тураевым в 90-х гг., которые и построили наиболее известные "квантовые" топологические инварианты 3-многообразий, которые можно считать точным определением континуального интеграла Виттена. Ряд результатов получен также Аксельродом-Зингером в 90-х гг. Топологические применения этих величин могли бы стать весьма значительными, если бы удалось глубже понять их связь с топологическими свойствами многообразий и с классической алгебраической топологией. 


\section{Новые идеи в теории Морса}

Взаимодействие с современной математической и теоретической физикой принесло ряд новых идей в топологические аспекты вариационного исчисления. Новиков, исходя из гамильтоновой факторизации систем классической механики (например, волчка в гравитационном поле или в идеальной жидкости), а также из теории движения заряженных частиц в топологически нетривиальном магнитном поле, начал в 1981 г. построение аналога теории Морса для замкнутых 1-форм вместо однозначных функций (функционал действия этих систем плохо определен, однако его вариация является замкнутой 1-формой на пространствах путей). Была дана классификация подобных локальных лагранжианов в многомерной теории поля (действие Весса-Зумино-НовиковаВиттена) и сформулировано условие "топологического квантования констант связи", вытекающее из требования, что фейнмановская амплитуда (т.е. экспонента от действия, умноженного на мнимую единицу) является однозначным функционалом. Тем самым вариация действия должна быть целочисленным классом 1-когомологий на пространстве полей. Это - альтернативная формулировка условия квантования Дирака для частицы в поле магнитного монополя, но сформулированная в рамках общепринятого теперь фейнмановского квантования вместо шредингеровского; это - удобная форма для многомерного обобщения. $\mathrm{K}$ подобным идеям в некоторых частных случаях пришли также Дезер-Джакив-Темплтон (1982) и Виттен (1983). Аналог теории Морса (теория "Морса-Новикова") была развита Новиковым и для 1-форм на конечномерных многообразиях. Оказалось, что в этом случае аналоги неравенств Морса для чисел критических точек ("неравенства Морса-Новикова") возникают при использовании когомологий с коэффициентами в так называемых "кольцах Новикова", простейшее из которых совпадает с кольцом лорановских рядов конечного порядка с целыми коэффициентами от одной переменной. Эта теория была развита Фарбером (1984), который доказал точность этих неравенств при определенных условиях (аналог теоремы Смейла). Построение теории Морса-Новикова на пространствах петель оказалось весьма трудным для частицы в магнитном поле: Новиков и Тайманов сформулировали и использовали "принцип перекидывания циклов" для многозначных функционалов, но обоснование было строго доказано ими лишь в некоторых частных случаях. 
Значительно позднее в симплектической топологии, начиная с работ Флоера (1988), посвященных так называемой гипотезе Арнольда о числе периодических траекторий неавтономных гамильтоновых систем, периодически зависящих от времени, на компактных симплектических многообразиях, фактически тоже появились многозначные функционалы, но это не сразу было осознано. Вариационный принцип Гамильтона на компактных симплектических многообразиях всегда приводит к ситуации типа Новикова: функционал действия всегда многозначен. В дальнейших работах МакДафф, Саломона и Хофера (начало 90-х гг.) эта теория была далеко развита. Мы не будем здесь обсуждать дальше весьма глубокие аспекты симплектической и контактной топологии, такие как инварианты Громова-Виттена, теорию ХофераЭлиашберга-Гивенталя, зеркальную симметрию и т.д.

В 1982 г. Виттен предложил оригинальный аналитический метод для доказательства обычных классических неравенств Морса. Его идея оказалась чрезвычайно плодотворной, но мы не будем здесь вникать в ее детали. Метод Виттена был применен Пажитновым (1987) к обсуждавшейся выше задаче Новикова об оценке чисел критических точек замкнутых 1-форм. Новиков сделал попытку применения этого метода и к незамкнутым формам (векторным полям). Атья и Ботт успешно применяли метод Виттена к голоморфным векторным полям, но этот предмет выходит из рамок данной статьи.

Был проведен ряд исследований для неодносвязных многообразий в 80-х гг., используя многообразия представлений фундаментальной группы - для целей теории Морса (Новиков, 1986), а также для теории узлов (Ле Тханг, начало 90-х гг.). Эта область имеет значительные перспективы, ряд топологов в последние годы уделили большое внимание изучению многообразий представлений.

\section{Новые идеи в топологии 80-х-90-х гг.: четырехмерные многообразия}

Сообщество физиков-теоретиков стало проявлять возрастающий интерес к идеям алгебраической топологии, начиная с 70-х гг. Стало ясно, что в природе имеется немало реальных явлений топологической природы, и особенно это касается квантовой физики. Всюду, где появляются нетривиальные феномены, связанные 
с магнитным полем или его аналогами - такими, как поля ЯнгаМиллса (дифференциально-геометрические связности в векторных расслоениях), обязательно появится топология. Всюду, где возникают поля сложного математического характера, с нетривиальными особенностями, появится топология. Подобные ситуации появляются в низкотемпературных фазах многих веществ, в жидких кристаллах и других. Мы упомянем здесь только идеи физиков, оказавшие большое обратное влияние на идеи самой топологии. В теории полей Янга-Миллса были обнаружены такие топологические феномены, как монополь Полякова-Т'Хоофта (1973), пока еще экспериментально не обнаруженный, и инстантон, открытый в работе Полякова-Шварца-Белавина-Тюпкина (1974), а также замечательное уравнение автодуальности, которому этот инстантон удовлетворяет, найденное в той же работе. Не обсуждая судьбу этих идей в физике, напомним, что в начале 80-х гг. Дональдсон нашел замечательное применение инстантонов и уравнения автодуальности в 4-мерной топологии: например, удалось доказать, что всякое 4-многообразие (замкнутое, односвязное) с положительной формой пересечений 2-циклов гомотопически эквивалентно связной сумме проективных плоскостей или им комплексно-сопряженных - т.е. геометрически реализуется только тривиальный набор положительных форм. Им были обнаружены различные гладкие структуры на некоторых 4-многообразиях. Этот замечательный факт опирается на глубокие результаты по качественной теории нелинейных эллиптических уравнений с частными производными - итог большой и очень трудной теории. Он опирается также на теорему Фридмана о гомеоморфизме 4-многообразий (выше). Еще со времен Кодаиры известны списки алгебраических поверхностей, где не очень сильно потрудившись, можно найти два односвязных 4-многообразия, построенные поразному, но имеющие изоморфные формы пересечений циклов. По Фридману, они на самом деле гомеоморфны. Однако на этих многообразиях различны многообразия инстантонов - решений уравнения автодуальности. Это и решает дело - они недиффеоморфны!

Позднее этого типа аргументы были сильно улучшены, было найдено более простое, гораздо легче исследуемое уравнение Зайберга-Виттена, позволившее сделать теоремы обоснования легкими и получить множество подобных результатов. Была доказана также гипотеза Тома о роде минимальной поверхно- 
сти, реализующей 2-цикл (Кронхаймер и Мровка, конец 90-х гг.). Финташел в середине 90-х гг. нашел красивую связь уравнений Зайберга-Виттена с полиномом Александера узлов при определенном способе построения многообразий. Он получил возможность эффективно вычислить эти инварианты в ряде примеров, ведущих к сильным результатам. Детали нового периода развития топологии мы отложим для специальных статей.

Мы не обсуждаем в этой статье такие области, как топология и геометрия слоений на многообразиях, теория особенностей, контактная и симплектическая топология и геометрия, тесно связанные с обсуждавшимися выше новыми методами топологии.

Целый ряд глубоких аспектов топологии неодносвязных многообразий также остался за пределами нашей статьи, включая теорию "фон Неймановских инвариантов" - аналога чисел Бетти и формулы индекса, неравенств Морса и кручения Радемайстера, развивавшуюся Атьей, Зингером, Новиковым, Шубиным, Лоттом, Громовым, Фарбером в 1980-х гг.

\section{Список литературы}

[1] Новиков С., Топология. 2-е изд. (русск.) - Москва-Ижевск, 2002; Novikov S. P., Gamkrelidze R. V. (eds.). Topology I. Part 1: General survey. (In English.) - Berlin: Springer-Verlag, 1996. (Encyclopaedia Math. Sci. V. 12.)

[2] Виро О., Фукс Д., “Топология II. Введение в теорию гомотопий" // Итоги науки и техники. Современные проблемы математики. Фундаментальные направления. 24 - М.: ВИНИТИ, 1988; Fuks D., Viro О. Topology II. (In English.) - New York: Springer-Verlag, 2003. (Encyclopedia Math. Sci.)

[3] Дубровин Б., Новиков С., Фоменко А., Современная геометрия. Т. I, II, III. - М.: Наука, 1998.

[4] Новиков С., Тайманов И., Современные геометрические структуры и поля. - М.: Изд-во Независимого Университета, 2004.

[5] Милнор Дж., Теория Морса. (Русск.) - М.: Мир, 1965.

[6] Милнор Дж., Теорема об h-кобордизме. (Русск.) - М.: Мир, 1970. 
[7] Понтрягин Л., "Гладкие многообразия и их применение в теории гомотопий" // Избранные научные труды. Т. 1. - М.: Наука, 1988. С. 548-677.

[8] Зейферт Г., Трельфалль В., Топология. (Русск.) - М.: ГОНТИ, 1938; 2-е изд. Ижевск: НИЦ "Регулярная и хаотическая динамика", 2001.

[9] Ботт Р., Ту Л., Дифференииалъные формы в алгебраической топологии. - М.: Наука, 1989.

[10] Атья М., Лекции по K-теории. (Русск.) - М.: Мир, 1967.

[11] Фукс Д., Фоменко А., Гутенмахер В., Гомотопическая топология. - М.: Изд-во МГУ, 1969. 


\section{Именной указатель}

Абель Н. (Abel N.) 5

Адамс Дж. (Adams J.)

$18,19,20,21,22,29$

Адем Дж. (Adem J.) 11, 12

Аксельрод C. (Axelrod S.) 35

Александер Дж. (Alexander J.)

$6,7,8,25,33,39$

Аппель K. (Appel K.) 31

Артин Э. (Artin E.) 8

Apф K. (Arf C.) 9, 15, 19

Атья M. (Atiyah M.)

$4,18,20,34,37,39$

Бакстер P. (Baxter R.) 33

Бар-Натан Д. (Bar-Natan D.) 33

Бельтрами Э. (Beltrami E.) 34

Бернулли Я. (Bernoulli J.) 21

Бетти Э. (Betti E.) 6, 16, 39

Бирман Дж. (Birman J.) 34

Борель А. (Borel A.) 11, 12

Ботт P. (Bott R.) 17, 18, 20, 37

Браудер В. (Browder W.)

16, 17, 19, 20, 24, 26

Браун М. (Brown M.) 25

Браун Э. (Brown E.) 17, 19, 22

Бpayep Л. (Brouwer L.) 6

Вайнбергер Ш. (Weinberger Sh.)

17,28

Вальдхаузен Ф. (Waldhausen F.)
Весс Дж. (Wess J.) 36

Вилкерсон K. (Wilkerson C.) 17

Вильсон В. (Wilson W.) 29, 34

Виттен Э. (Witten Е.) 4, 22, 34, $35,36,37,38,39$

By B.-T. (Wu W.-T.) 13

Вьеторис Л. (Vietoris L.) 7

Гамильтон У. (Hamilton W.) 37

Гaycc K. (Gauss C.) 5

Голдман B. (Goldman W.) 32

Гриффитс Ф. (Griffiths Ph.) 29

Гротендик А. (Grothendieck A.) 4, 20, 26

Гуревич B. (Hurewicz W.) 7

Дезер C. (Deser S.) 36

Делинь П. (Deligne P.) 4, 29

Ден M. (Dehn M.) 6, 30

Джакив P. (Jackiw R.) 36

Джонс У. (Jones W.) 4, 33, 34

Дирак П. (Dirac P.) 28, 36

Дольд A. (Dold A.) 14, 21, 22

Дональдсон C. (Donaldson S.)

\section{4,38}

Дьедонне ЖK. (Dieudonné J.) 9

Зайберг H. (Seiberg N.) 38, 39

Зариский O. (Zariski O.) 26

Зибенман Л. (Siebenmann L.) 27

Зингер И. (Singer I.)

20, 34, 35, 39 
Зумино Б. (Zumino В.) 36

Калаби Е. (Calabi E.) 22, 23 ван Кампен Э.

(van Kampen E.) 8

Картан А. (Cartan H.) 11, 12

Картан Э. (Cartan E.) 7, 8, 11

Кассон A. (Casson A.) 27, 31

Кауфман Л. (Kaufmann L.) 33

Квиллен Д. (Quillen D.)

$$
\text { 4, 22, } 29
$$

Квин Ф. (Quinn F.) 27

Кельвин У. (Kelvin W.) 5, 30, 34

Кервер M. (Kervaire M.)

$$
14,15,18,24
$$

Кирби Р. (Kirby R.) 27

Кодаира К. (Kodaira K.) 4, 38

Кон A. (Connes A.) 28

Коннер П. (Conner P.) 21

Коши О. (Cauchy A.) 5

Kрек M. (Kreck M.) 28

Кронхаймер П.

(Kronheimer P.) 39

Кэли А. (Cayley А.) 10

Лазар M. (Lazard M.) 22

Ландвебер П. (Landweber P.) 21,22

Лаплас П. (Laplace P.) 34

Лаусон Г. (Lawson H.) 28

Лашоф P. (Lashof R.) 27

Левин Ж. (Levine J.) 17

Лepe Ж. (Leray J.) 11

Лe T. (Le T.) 37

Лефшец C. (Lefschetz S.)

$$
6,7,8,9
$$

Ли С. (Lie S.) 11, 17, 29, 32, 34

Лин K. (Lin X.) 34

Листинг И. (Listing J.) 5

Лотт Дж. (Lott J.) 39

Люстиг Г. (Lusztig G.) 17, 28
Магнус В. (Magnus W.) 8

Мадсен И. (Madsen I.) 27

Мазур Б. (Masur B.) 25

МакДафф Д. (McDuff D.) 37

Маклейн C. (MacLane S.) 10, 12

Максвелл Дж. (Maxwell J.) 5

Мамфорд Д. (Mumford D.) 4

Манкрес Дж. (Munkres J.) 24

Масси У. (Massey W.) 10

Маховальд M. (Mahowald M.) 18

Миллс P. (Mills R.) 34, 38

Милнор Дж. (Milnor J.) 4, 14, 15, 17, 18, 19, 21, $24,25,30$

Мильгрэм P. (Milgram R.) 27

Мислин Г. (Mislin G.) 29

Мойс Э. (Moise E.) 25

Морава Дж. (Morava J.) 29

Морган Дж. (Morgan J.) 29

Mopc M. (Morse M.) 5, 7, 11, 15, $16,17,36,37,39$

мровка T. (Mrowka T.) 39

Мурасуги К. (Murasugi K.) 33

Мюллер В. (Muller W.) 34

Нейман Дж. (Neumann J.) 39

Нётер Э. (Noether E.) 7

Нётер $\Phi$. (Noether F.) 20

Нильсен Дж. (Nielsen J.) 8

Нишида Г. (Nishida G.) 29

Отал Ж.-П. (Otal J.-P.) 32

Папакирьякопулос С.

(Papakyriakopoulos C.) 30

Петерсон Ф. (Peterson F.) 19, 22 Пуанкаре A. (Poincaré H.) 5, 6, $7,8,15,23,31,32,33$

Рабин P. (Rabin R.) 16 
Равенел Д. (Ravenel D.) 29

Райдемайстер K.

(Reidemeister K.)

8, 23, 25, 28, 34, 39

де Рам Ж. (de Rham G.) 7, 11

Рей Д. (Ray D.) 34

Риман Б. (Riemann B.) 5, 13, 20

Риччи Г. (Ricci G.) 33

Розенберг Дж.

(Rosenberg J.) 28

Ротенберг M.

(Rothenberg M.) 27

Pox Г. (Roch G.) 13, 20

Рубинштейн И.

(Rubinstein I.) 31

Саймонс Дж. (Simons J.) 34

Саломон Д. (Salomon D.) 37

Cepp ЖК.-П. (Serre J.-P.) 4, 11, $12,13,18$

Серф Ж. (Serf J.) 24

Сигал Г. (Segal G.) 34

Смейл C. (Smale S.) 4, 15, 16, 27 , 31,36

Смит П. (Smith P.) 9

Спеньер Э. (Spanier E.) 8

Стинрод Н. (Steenrod N.) 10, 11,

$12,13,17,18,19,20,21$

Столлингс Дж. (Stallings J.) 15

Сулливан Д. (Sullivan D.) 17, 26, 27, 28, 29

Сян В.-Ч. (Hsiang W.-Ch.) 27

Телеман C. (Teleman S.) 28

Темплтон C. (Templeton S.) 36

Тёрстон У. (Thurston W.) 4, 32

Тода X. (Toda H.) 18, 21

Том P. (Thom R.) 4, 11, 12, 13, $14,15,16,19,20,24,25$, 29,38

Томпсон A. (Thompson A.) 31
T’Хоофт Ж. (T'Hooft G.) 38

Тейт П. (Tait Р.) 5, 33

Уайтхед Дж. (Whitehead G.) 20

Уайтхед Дж. (Whitehead J.)

$8,10,11,23,28,30,34$

Уитни X. (Whitney H.)

$8,9,13,23,26,29$

Уолл Ч. (Wall Ch.) 17, 19

Уоллес А. (Wallace A.) 15

Фарадей М. (Faraday M.) 5

Фаррел Ф. (Farrel F.) 27

Филдс Дж. (Fields J.) 4

Финташел P. (Fintushel R.) 39

Флоер A. (Floer A.) 37

Флойд Э. (Floyd E.) 21

Фрейденталь Х.

(Freudenthal H.) 7

Фридман M. (Freedman M.)

$4,23,31,38$

Хакен В. (Haken W.) 31, 32

Хёфлигер А. (Haefliger А.) 16

Хилтон П. (Hilton P.) 12, 29

Хирцебрух $\Phi$. (Hirzebruch F.)

$13,14,15,18,20,21$,

26,28

Хирш М. (Hirsch М.) 16, 24

Хопф X. (Hopf H.) 7, 10, 11, 12, $14,17,18,21,22,30,34$

Xoфep X. (Hofer H.) 37

Чен K.-T. (Chen K.-T.) 29

Чепмен Т. (Chapman T.) 28

Черн Ш.-Ш. (Chern Sh.-Sh.)

$$
9,21,22,34
$$

Чex Э. (Chech E.) 7, 8

Чигер Дж. (Cheeger J.) 34

Шейнсон Дж. (Shaneson J.) 17

Шенфлис А. (Schoenflies A.) 25 
Штифель Э. (Stiefel Е.)

9, 13, 23, 26

Штольц C. (Stolz S.) 28

Эдвардс P. (Edwards R.) 25

Эйленберг C. (Eilenberg S.) $7,10,12,20$

Эйлер Л. (Euler L.) 5

Экман Б. (Eckmann B.) 12

Якоби К. (Jacobi C.) 5

Янг Ч. (Yang Ch.) 33, 34, 38

Яних K. (Jänich K.) 35

Яо Ш.-T. (Yau Sh.-T.) 4, 22, 23

$$
* \quad * \quad *
$$

Адян С. 16

Александров П. 7

Арнольд В. 23, 37

Белавин А. 38

Бокштейн М. 10, 12

Ботвинник Б. 19

Бухштабер В. 21, 22, 29

Васильев В. 33

Вершинин В. 19

Виро О. 35

Воеводский В. 4

Гамкрелидзе Р. 21

Гельфанд И. 20

Гивенталь А. 37

Громов М. 28, 37, 39

Гусейн-Заде С. 22

Дынников И. 32

Ивановский Л. 19

Каспаров Г. 17, 28

Колмогоров А. 8

Концевич М. 4, 33

Кочман C. 19
Кричевер И. 22

Лобачевский Н. 32

Люстерник Л. 7

Марков А. (мл.) 8, 16, 33

Матвеев С. 31

Мищенко А. 17, 22, 28

Мусхелишвили Н. 20

Набутовский А. 17

Новиков П. 16

Новиков С. 4, 14, 15, 16, 17, 18, $19,20,21,22,23,24,26$, $27,28,29,35,36,37,39$

Ошанин С. 22

Пажитнов А. 37

Перельман Г. 33

Поляков А. 38

Понтрягин Л. 7, 8, 9, 10, 13, 14, $15,16,17,20,22,23,24$, $26,27,28$

Попов В. 34

Постников М. 10, 17

Решетихин Н. 35

Рохлин В. 13, 14, 15, 19, 27, 35

Семенов-Тян-Шанский М. 22

Тайманов И. 36

Тураев В. 33, 35

Тюпкин Ю. 38

Фаддеев Д. 10

Фаддеев Л. 22, 34

Фарбер М. 36, 39

Чернавский А. 27

Шварц А. 15, 34, 38

Шнирельман Л. 7

Штанько М. 25

Шубин М. 39

Элиашберг Я. 37 


\section{Содержание}

Алгебраическая топология 3

С.П. Новиков

Исторический очерк: топологические идеи до XIX века . . . . . . . . . . . . . . . . . . . 4

Топологические идеи в XIX веке . . . . . . . . . . . 5

Топология в ХХ веке: очерк до 1945 г. . . . . . . . . 6

Алгебраическая топология в ХХ веке: 1945-1955 гг. 10

А.Т. в период 1956-1970 гг. . . . . . . . . . . . . . . . 14

Непрерывные гомеоморфизмы в топологии . . . . . 23

Маломерная и гиперболическая топология . . . . . 30

Новые идеи в теории Морса . . . . . . . . . . . . . . 36

Новые идеи в топологии 80-х-90-х гг.: четырехмерные многообразия . . . . . . . . . . . 37

Список литературы . . . . . . . . . . . . . . . . . . . 39

Именной указатель . . . . . . . . . . . . . . . . . . . 41 